\title{
Capacity and Needs Assessment for Establishing a Syndromic Surveillance System in Rural China: A Mixed Study
}

\author{
Tao Tao*1, Qi Zhao ${ }^{1}$, Huijian Cheng ${ }^{2}$, Weirong Yan ${ }^{3,4}$, Hengjin Dong ${ }^{5,6}$ and Biao $\mathrm{Xu}^{1}$ \\ ${ }^{1}$ School of Public Health, Fudan University, Shanghai, China; ${ }^{2} J i a n g x i$ Provincial Center for Disease Control and Prevention, \\ Nanchang, China; ${ }^{3}$ Division of Global Health (IHCAR), Department of Public Health Sciences, Karolinska Institutet, Stockholm, \\ Sweden; ${ }^{4}$ Department of Epidemiology and Biostatistics, School of Public Health, Tongji Medical College, Huazhong University of \\ Science and Technology, Wuhan, China; ${ }^{5}$ nstitute of Public Health, Heidelberg University, Heidelberg, Germany; ${ }^{6}$ Center for Health \\ Policy Studies, School of Public Health, Zhejiang University School of Medicine, Hangzhou, China
}

\section{Objective}

Before the start of ISSC project, a pilot investigation was carried out among the candidate surveillance units (health care facility, pharmacy and primary school) and related stakeholders to assess their capacity and potential needs with regard to the implementation of ISSC system, so as to design customized capacity building and training strategies.

\section{Introduction}

Syndromic surveillance has great advantages in promoting the early detection of infectious disease outbreak and enabling the real-time tracking of on-going epidemics. However, establishing a syndromic surveillance system required huge investment in money, information system, manpower and capacity building activities, which remains a big challenge in resource-limited areas. Funded by European Union's 7th Framework Programme, a syndromic surveillance system named "ISSC" was prepared to be built and incorporated with the existing case report system in rural Jiangxi Province of China.

\section{Methods}

Two rural counties of Jiangxi Province (Yongxiu County and Fengxin County), where the ISSC system was to be implemented, were chosen as our study sites. Both quantitative and qualitative methods were introduced and mixed in this study. First, we conducted a unit-based questionnaire survey among all health care facilities, pharmacies and primary schools within the township and village levels of the two counties, to investigate the basic characteristics, manpower allocation, and feasibility of data collection in these candidate surveillance units. Then, focus group discussions (FGDs), personal interviews and an individual questionnaire survey were carried out among health workers, pharmacy staffs and school health teachers to obtain their perception and attitude towards the implementation of ISSC surveillance system.

\section{Results}

The outpatient $\log$ in health care facility, the drug sale record in pharmacy and the school absenteeism report in primary school could be used and modified as syndromic data sources for ISSC surveillance system. In each township hospital, village health station and primary school, there was at least one person in charge of the infectious disease control and prevention job, but in pharmacies there was no person assigned with disease control or surveillance work. Results of the individual survey showed that although most of doctors investigated reported they were capable to accomplish surveillance tasks in ISSC project, a considerable part of them (32.5\% in Fengxin County and $51.3 \%$ in Yongxiu County) considered the workload of data reporting for ISSC system was heavy. According to FGDs carried out in different surveillance units, the lack of manpower and spare time among rural workers remained the biggest challenge in the future implementation of ISSC system. Some participants indicated that in most primary school and some health care facilities, the recording of outpatient $\log$ and school absenteeism report (candidate data sources for ISSC system) was incomplete or inconsistent due to the lack of rigid regulations in rural areas. In addition, a part of participants from pharmacy and primary school could not understand the objective and significance of syndromic surveillance system, and showed a low willingness of cooperation with regard to ISSC implementation.

\section{Conclusions}

The existing infrastructures, data sources and human resources related to health care and national disease control networks could be utilized for establishing the ISSC syndromic surveillance system. However, potential obstacles lied in the data collection and reporting, data quality control, acceptability of local workers and long-term sustainability. Training on computer techniques, operations of electronic surveillance system, and the knowledge on infectious disease control were of great importance. Effective incentive measures should be introduced along with the implementation of ISSC system to ensure the routine collection of high-quality surveillance data.

\section{Keywords}

syndromic surveillance; capacity; implementation

\section{Acknowledgments}

This study was funded by European Union's Seventh Framework Programme ([FP7/2007-2013] [FP7/2007-2011]) under grant agreement no. [241900].

\section{*Tao Tao}

E-mail: ttsuper2000@hotmail.com 